\title{
AN ERGODIC THEOREM FOR A NONCOMMUTATIVE SEMIGROUP OF LINEAR OPERATORS
}

\author{
J. E. L. PECK
}

Introduction. The question of ergodicity of a semigroup of bounded linear operators on a Banach space has been reduced, by Alaoglu and Birkhoff [1], ${ }^{1}$ Day [2, 3], and Eberlein [4], to the study firstly of the ergodicity of the semigroup itself and secondly, of the ergodicity of each element of the Banach space with respect to this ergodic semigroup. In the case of a bounded and commutative semigroup operating on a reflexive Banach space, the ergodicity has been established [2, Corollary 6], [4, Corollary 5.1, Theorem 5.5]. For noncommutative semigroups, Alaoglu and Birkhoff have proved ergodicity by restricting the Banach space [1, Theorem 6$]$. A general discussion of the noncommutative case is given by Day [3].

The following is an attempt to prove ergodicity, in the strong topology, for a certain noncommutative case, where we use a weakened form of the commutative law. Our method uses a fixed point theorem of Kakutani [5], and a theorem about the group of cluster points of a directed subsemigroup, which we have developed elsewhere [6].

It should be observed, however, that the theorem which is proved here, though being similar to, is not a generalization of the results mentioned above. In fact it is apparently independent of them, because we postulate here a compactness in the strong topology of operators; whereas in the analogous theorems of Day and Eberlein, a kind of weak compactness is used, by considering bounded semigroups acting on reflexive spaces, a natural legacy from the study of Hilbert space. However our theorem is a generalization of a theorem of Yosida [7, Theorem 2].

Preliminary theorems. In this section we prove a theorem in a slightly more general form than we shall require. We need the following notions. A linear space over the field of complex numbers is a linear topological space [8] if it has a separated topology [13], which is such that all operations are continuous. More precisely, there is a fundamental system of neighborhoods of the zero element $\left\{V_{\alpha} \mid \alpha \in \Delta\right\}$, with the following properties:

(1) The intersection of all $V_{\alpha}$ is the zero element.

Presented to the Society, April 29, 1950; received by the editors June 22, 1950.

${ }^{1}$ Numbers in brackets refer to the references at the end of the paper. 
(2) Given $\alpha, \beta \in \Delta$ we may find $\gamma \in \Delta$ such that $V_{\gamma} \subseteq V_{\alpha} \cap V_{\beta}$.

(3) For any $\alpha \in \Delta$ there is a $\beta \in \Delta$ with $V_{\beta}+V_{\beta} \subseteq V_{\alpha}$.

(4) For any $\alpha \in \Delta$ there is a $\beta \in \Delta$ such that if $c$ is a complex number and $|c| \leqq 1$, then $c V_{\beta} \subseteq V_{\alpha}$.

(5) For any element $s$ of the space and any $\alpha \in \Delta$ there is a positive real number $r$ such that $s \in r V_{\alpha}$.

Also, this system is locally convex if in addition

(6) For any $\alpha \in \Delta$ there is a $\beta \in \Delta$ such that $V_{\beta} \subseteq V_{\alpha}$ and $V_{\beta}$ is convex.

A subset $\mathcal{S}$ of a linear topological space $\mathcal{L}$ is called bounded if to each $\alpha \in \Delta$ there is a complex scalar $c$ such that $S \subseteq c V_{\alpha}$, and it is called totally bounded if to each $\alpha \in \Delta$ there is a finite set $s_{1}, \cdots, s_{n}$ $\in \mathcal{L}$ such that $\delta \subseteq \bigcup_{i=1}^{n}\left[s_{i}+V_{\alpha}\right]$. We observe that we may restrict the finite set to lie in $\delta$ [10]. A linear topological space is called boundedly complete [9] if every bounded, fundamental directed set $[11]$ is convergent. Finally a linear transformation $\phi$ of a linear topological space to itself is called congruent [5] if $\phi V_{\alpha}=V_{\alpha}$ for all $\alpha \in \Delta$.

We shall use a fixed point theorem of Kakutani [5] which appears in the literature without proof. With the permission of the author we reproduce the proof here, for the sake of completeness. This proof makes use of Maak's Lemma [12] which we quote here.

THEOREM 1 (MAAK). If the set $M$ is partitioned into $n$ subsets in two different ways, $M=A_{1} \cup \ldots \cup A_{n}=B_{1} \cup \ldots \cup B_{n}$, so that any $r$ of the $A_{i}$ 's contain at most $r$ of the $B_{j}$ 's, then there is a common representative system. That is, the subscripts may be reordered in such a way that $A_{i} \cap B_{i} \neq \varnothing, i=1, \cdots, n$.

TheOREM 2 (KAKUTANI). Let $\mathcal{L}$ be a locally convex linear topological space. Let $\mathrm{B}$ be a compact, convex subset of $\mathcal{L}$, and let $\Phi$ be any set of linear (more generally affine) congruent transformations of $\mathcal{L}$ into itself, each of which takes $B$ onto itself. Then there is an element $p \in \mathcal{B}$ which is such that $\phi p=p$ for all $\phi \in \Phi$.

Proof. We may assume that all $\left\{V_{\alpha} \mid \alpha \in \Delta\right\}$ are convex neighborhoods and that $V_{\alpha}=-V_{\alpha}$. Since $B$ is compact, then for each $\alpha \in \Delta$ we may find a finite set $b_{1}, \cdots, b_{n} \in \mathcal{B}$ such that $B$ is contained in $V_{\alpha}\left(b_{1}\right) \cup \ldots \cup V_{\alpha}\left(b_{n}\right)$, where $V_{\alpha}(b)=V_{\alpha}+b$. We may suppose also that $n$ is chosen so that it is minimal. Then because $\phi$ is congruent, we have

$$
\mathbb{B}=\phi \mathcal{B} \subseteq \phi V_{\alpha}\left(b_{1}\right) \cup \ldots \cup \phi V_{\alpha}\left(b_{n}\right)=V_{\alpha}\left(\phi b_{1}\right) \cup \ldots \cup V_{\alpha}\left(\phi b_{n}\right) .
$$

Put 
$A_{1}=V_{\alpha}\left(b_{1}\right) \cap \mathcal{B}, \quad A_{k}=\left[V_{\alpha}\left(b_{k}\right)-\bigcup_{i=1}^{k-1} A_{i}\right] \cap \mathbb{B}, \quad k=2, \cdots, n$, $B_{1}=V_{\alpha}\left(\phi b_{1}\right) \cap \mathcal{B}, \quad B_{k}=\left[V_{\alpha}\left(\phi b_{k}\right)-\bigcup_{i=1}^{k-1} B_{i}\right] \cap \mathbb{B}, \quad k=2, \cdots, n$.

Then because of the choice of $n$, none of these sets is vacuous. We thus have two partitions $A_{1} \cup \ldots \cup A_{n}=B_{1} \cup \ldots \cup B_{n}=\mathbb{B}$ which satisfy the postulates of Theorem 1. In fact, if there are $r$ of the $A$ 's which contain $r+1$ of the $B$ 's, then replacing these $B$ 's by the $A$ 's which contain them, we are led to a covering of $B$ with $n-1$ elements, contradicting the choice of $n$. Thus using the conclusions of Theorem 1 , it is possible to reorder the set $b_{1}, \cdots, b_{n}$, say to $c_{1}, \cdots, c_{n}$, such that $V_{\alpha}\left(b_{i}\right) \cap V_{\alpha}\left(\phi c_{i}\right) \neq \varnothing, i=1, \cdots, n$.

Put

$$
a_{\alpha}=\left[b_{1}+\cdots+b_{n}\right] / n=\left[c_{1}+\cdots+c_{n}\right] / n \text {. }
$$

Then

$$
\begin{gathered}
\phi a_{\alpha}=\left[\phi b_{1}+\cdots+\phi b_{n}\right] / n=\left[\phi c_{1}+\cdots+\phi c_{n}\right] / n, \\
a_{\alpha}-\phi a_{\alpha}=\left[\left(b_{1}-\phi c_{1}\right)+\cdots+\left(b_{n}-\phi c_{n}\right)\right] / n \\
\in\left[2 V_{\alpha}+\cdots+2 V_{\alpha}\right] / n \subseteq 2 V_{\alpha} .
\end{gathered}
$$

Thus to each $\alpha$ we associate a point $a_{\alpha}$ independently of $\phi$, which is moved a small distance by $\phi$. This directed set $\left\{a_{\alpha} \mid \alpha \in \Delta\right\}$, being in a compact space, will have at least one cluster point [14]. Call it $p$. We prove that $\phi p=p$. In fact, given $\beta \in \Delta$, we may find $\alpha>\beta$ such that $a_{\alpha} \in V_{\beta}(p)$, whence $\phi a_{\alpha} \in V_{\beta}(\phi p)$. Thus

$$
\begin{gathered}
\phi p-p=\phi p-\phi a_{\alpha}+\phi a_{\alpha}-a_{\alpha}+a_{\alpha}-p \\
\in V_{\beta}+2 V_{\beta}+V_{\beta} \subseteq 4 V_{\beta} .
\end{gathered}
$$

But since $\beta$ was arbitrary, this proves that $\phi p=p$.

Theorem 3. Let $\mathcal{E}$ be a boundedly complete, locally convex, linear topological space, which is also an abstract algebra, where the new operation satisfies the conditions

(1) if $\mathcal{A}$ is a totally bounded subset of $\mathcal{E}$, then given $\alpha \in \Delta$ we may find $\beta \in \Delta$ such that $\mathcal{A} V_{\beta} \subseteq V_{\alpha}$, and

(2) If $A$ is an element of $\mathcal{E}$ then given $\alpha \in \Delta$ we may find $\beta \in \Delta$ such that $V_{\beta} A \subseteq V_{\alpha}$.

Let $\mathcal{G}$ be a totally bounded, multiplicative group in $\mathcal{E}$, then there is an element $p \in \mathrm{Cl}($ con $G$ ) (the closed convex set generated by $G$ ) which is such that $g p=p=p g$ for all $g \in G$, and $p^{2}=p$.

Proof. Let $\mathcal{L}$ be the set of all bounded functions defined on $G$ with values in $\mathcal{E}$. Then $\mathcal{L}$ is a linear space in the usual way $[10$, Defi- 
nition 11]. In $\mathcal{L}$ we introduce the uniform function topology, generated by the topology of $\mathcal{E}$, that is, $\left\{V_{\alpha}^{\prime} \mid \alpha \in \Delta\right\}$ is a system of neighborhoods of the zero element of $\mathcal{L}$, where $V_{\alpha}^{\prime}=\{L \mid L \in \mathcal{L}$, $\left.L(G) \subseteq V_{\alpha}\right\}$ and $\left\{V_{\alpha} \mid \alpha \in \Delta\right\}$ is the system of convex neighborhoods of the zero element of $\varepsilon$ which are such that $V_{\alpha}=-V_{\alpha}$. Under this topology $\mathcal{L}$ becomes a locally convex, linear topological space [10, Theorem 17]. This space is also boundedly complete, for if we suppose that $\left\{L_{\gamma} \mid \gamma \in \Gamma\right\}$ is a bounded fundamental directed set in $\mathcal{L}$, then for a fixed $g \in G, L_{\gamma}(g)$ is a fundamental directed set in $\varepsilon$, which is bounded, the same bound serving for all $\mathrm{g}$. But $\varepsilon$ is boundedly complete, so there is an element $L_{\infty}(g)$ in $\varepsilon$ such that $L_{\gamma}(g) \rightarrow L_{\infty}(g)$ in $\varepsilon$. The function $L_{\infty}$ thus defined on $G$ to $\varepsilon$ is bounded because $L_{\gamma}(g)$ is bounded uniformly with respect to $\gamma$ and $g$. Finally $L_{\infty}$ is constructed so that $L_{\gamma} \rightarrow L_{\infty}$ in $\mathcal{L}$.

Let $\phi_{a}$ be the transformation of $\mathcal{L}$ into itself, defined by $\left(\phi_{a} L\right) g$ $=L(a g)$. The set $\left\{\phi_{a} \mid a \in G\right\}$ will be denoted by $\Phi$. Each $\phi_{a}$ is a linear, congruent transformation of $\mathcal{L}$ into itself.

Let $I$ be the function in $\mathcal{L}$ which is such that $I(g)=g$ (the identity). It is certainly bounded because $G$ is totally bounded $[10$, Theorem 10].

Put $\mathbb{B}=\mathrm{Cl}(\operatorname{con}(\Phi I))$, so that $\mathbb{B}$ is the closed, convex set in $\mathcal{L}$ generated by the set of all left translates of the function $I$. We shall prove that $\mathcal{B}$ is compact. To do this it is sufficient to establish that the set $\Phi I$ is totally bounded in $\mathcal{L}$, for then $[10$, Theorems $10,11,12]$ $\mathrm{Cl}(\operatorname{con}(\Phi I))$ is totally bounded and bounded, and therefore as a closed bounded subset of a boundedly complete space, it is complete. But a totally bounded, complete uniform space is compact $[13, \mathrm{p}$. 111].

Thus we have to show that for any given $\alpha \in \Delta$, we may find a finite set $a_{1}, \cdots, a_{n} \in G$ such that to any $a \in G$, there is an $a_{j}$, $1 \leqq j \leqq n$, such that $I(a g)-I\left(a_{j} g\right)=a g-a_{j} g \in V_{\alpha}$ for all $g \in G$. First we choose $V_{\gamma}$ by hypothesis (1) so that $G V_{\gamma} \subseteq V_{\alpha} / 3$, because $G$ is totally bounded. Also we may choose $g_{1}, \cdots, g_{m} \in G$ so that $G$ $\subseteq \bigcup_{i=1}^{m}\left[g_{i}+V_{\gamma}\right]$, and then choose $V_{\delta}$ so that $V_{\delta} g_{i} \subseteq V_{\alpha} / 3,1 \leqq i \leqq m$, by hypothesis (2). Finally we choose $a_{1}, \cdots, a_{n} \in G$ so that $G$ $\subseteq \mathrm{U}_{j=1}^{n}\left[a_{j}+V_{\delta}\right]$. Then for any given $a \in G$, we may find an $a_{j}, 1 \leqq j \leqq n$, so that by using a suitable $g_{i}$ we have that

$$
\begin{aligned}
a g=a_{j} g & =a g-a g_{i}+a g_{i}-a_{j} g_{i}+a_{j} g_{i}-a_{j} g \\
& \in a V_{\gamma}+V_{\delta} g_{i}+a_{j} V_{\gamma} \\
& \subseteq\left[V_{\alpha}+V_{\alpha}+V_{\alpha}\right] / 3 \\
& \subseteq V_{\alpha} .
\end{aligned}
$$


Similarly we may define $\phi_{a}^{\prime}$ to be the congruent transformation $\left(\phi_{a}^{\prime} L\right) g=L(g a), \Phi^{\prime}=\left\{\phi_{a}^{\prime} \mid a \in G\right\}$ and $\mathcal{B}^{\prime}=\mathrm{Cl}\left(\right.$ con $\left.\left(\Phi^{\prime} I\right)\right)$. The closed, convex set $B^{\prime}$ will be compact if the set $\Phi^{\prime} I$ is totally bounded, and this is the case if given $\alpha \in \Delta$ we may find $a_{1}, \cdots, a_{n} \in G$ so that for any $a \in G, g a-g a_{j} \in V_{\alpha}$ for some $a_{j}$ and all $g$. But this is easier to establish than the previous case because, choosing $V_{\beta}$ so that $G V_{\beta} \subseteq V_{\alpha}$ and then $a_{1}, \cdots, a_{n}$ so that $G \subseteq \bigcup_{j=1}^{n}\left[a_{j}+V_{\beta}\right]$, then for any given $a \in G$ we may find an $a_{j}, 1 \leqq j \leqq n$, so that $g a-g a_{j} \in g V_{\beta}$ $\subseteq V_{\alpha}$.

We now observe that $\phi_{a} \mathcal{B}=\mathscr{B}$ and $\phi_{a}^{\prime} \mathcal{B}^{\prime}=\mathscr{B}^{\prime}$ for all $a \in G$ because $\phi_{a}\left[\phi_{b} I\right]=\phi_{b a} I \in \mathscr{B}$ and therefore $\phi_{a}$ con $(\Phi I) \subseteq \mathcal{B}$. Since $\phi_{a}$ is congruent and therefore continuous, $\phi_{a} B \subseteq B=\phi_{a^{-1}} \phi_{a} B \subseteq \phi_{a} \mathcal{B}$. Similarly with $\mathcal{B}^{\prime}$.

We now have all the hypotheses of Theorem 2, which we apply first to $B$ to obtain a function $P \in \mathbb{B}$ such that $\phi_{a} P=P$ for all $a \in G$. That is, $P(a g)=P(g)$, which proves that $P(g)$ is a constant, which we shall denote by $p$. Also $p \in \mathrm{Cl}(\operatorname{con} G)$. Since $P$ is in $\mathbb{B}$, then

$$
p=P(g)=\lim _{\alpha} \sum_{n=1}^{n_{\alpha}} \lambda_{\alpha, n} a_{\alpha, n} g, \quad \lambda_{\alpha, n} \geqq 0, \quad \sum_{n=1}^{n_{\alpha}} \lambda_{\alpha, n}=1,
$$

uniformly in $g$, whence using hypothesis (2)

$$
p=P(g)=P(g a)=p a,
$$

for all $a \in G$.

Similarly, applying the theorem to $\mathscr{B}^{\prime}$ and $\Phi^{\prime}$, we obtain a $p^{\prime}$ in $\mathrm{Cl}$ (con $G$ ) so that $p^{\prime}=a p^{\prime}$ for all $a \in G$. Taking the directed limit of convex sums gives us that $p=p p^{\prime}=p^{\prime}$, so that $p g=p=g p$ for all $g \in G$, and $p^{2}=p$.

Ergodicity. We apply the results of the previous section to obtain an ergodic theorem. Let $\varepsilon$ be the set of linear bounded operators acting on a Banach space $X$, in the strong topology of operators. That is, if we put $\alpha=\left\{x_{1}, \cdots, x_{n} ; \epsilon\right\}, x_{1}, \cdots, x_{n} \in X$ and $\epsilon>0$, and denote the set of all $\alpha$ by $\Delta$, then if we write

$$
V_{\alpha}=\left\{T|T \in \mathcal{E},| T x_{i} \mid<\epsilon, i=1, \cdots, n\right\},{ }^{2}
$$

the family $\left\{V_{\alpha}^{\prime} \mid \alpha \in \Delta\right\}$ is a fundamental system of convex neighborhoods of the zero operator in the strong topology.

It is easy to verify that $\varepsilon$ is a locally convex, linear topological space. Also $\mathcal{E}$ is boundedly complete, because if $\left\{T_{\gamma} \mid \gamma \in \Gamma\right\}$ is a bounded fundamental directed set in $\varepsilon$, then by the principle of uniform boundedness [15, Theorem 2.12.2] there is an $M>0$ such

\footnotetext{
${ }^{2}$ We use the notation || for the norm in a Banach space.
} 
that $\left|T_{\gamma}\right| \leqq M$ for all $\gamma \in \Gamma$. But $\left\{T_{\gamma} x \mid \gamma \in \Gamma\right\}$ is a fundamental directed set in $X$ which is complete, so we may find $T_{\infty} x$ so that $T_{\gamma} x$ $\rightarrow T_{\infty} x$ in $X$. It is a routine matter to verify that $T_{\infty}$ is a linear operator, $\left|T_{\infty}\right| \leqq M$, and $T_{\gamma} \rightarrow T_{\infty}$ in $\varepsilon$.

We know also that $\varepsilon$ is an abstract algebra satisfying the postulates (1) and (2) of Theorem 3, because: (1) if $\mathcal{A}$ is a totally bounded subset of $\varepsilon$, then it is bounded and therefore bounded in norm, that is, $|T| \leqq M$ for all $T$ in $\mathcal{A}$. Thus if $\alpha=\left\{x_{1}, \cdots, x_{n} ; \epsilon\right\}$ put $\beta$ $=\left\{x_{1}, \cdots, x_{n} ; \epsilon / M\right\}$, from which we have that $\mathcal{A} V_{\beta} \subseteq V_{\alpha} ;$ and (2) if $\alpha=\left\{x_{1}, \cdots, x_{n} ; \epsilon\right\}$, putting $\beta=\left\{T x_{1}, \cdots, T x_{n} ; \epsilon\right\}$ ensures that $V_{\beta} T \subseteq V_{\alpha}$.

We shall adopt the following definitions due to Eberlein [4]. A semigroup $\mathcal{S}$ in $\varepsilon$ is ergodic if there is a directed set of operators $\left\{T_{\gamma} \mid \gamma \in \Gamma\right\}$ in $\varepsilon$ with the properties:

(a) $T_{\gamma} x \in \mathrm{Cl}$ (con $\mathrm{S}$ ) $x$ for all $\gamma \in \Gamma$ and all $x \in X$,

(b) there is an $M>0$ such that $\left|T_{\gamma}\right| \leqq M$ for all $\gamma \in \Gamma$,

(c) if $T \in \mathcal{S}$, then $\lim _{\gamma}\left(T T_{\gamma}-T\right)=0, \lim _{\gamma}\left(T_{\gamma} T-T\right)=0$, where the limits are in the strong topology.

Also, an element $x \in X$ is ergodic with respect to this ergodic semigroup if there exists a $y=T_{\infty} x \in X$ such that $y \in \mathrm{Cl}((\operatorname{con} \delta) x)$ and $S y=y$ for all $S \in \mathcal{S}$.

We shall make use of the following theorems which have been developed elsewhere [6].

TheOREM 4. If $\mathcal{T}$ is a compact topological semigroup [15], and if $\mathcal{S}$ is a subsemigroup of $\tau$ which satisfies the pseudo-commutative conditions

(i) if $S_{1}, S_{2} \in \mathcal{S}$, then there exist $S_{3}, S_{4} \in \mathcal{S}$ such that $S_{1} S_{3}=S_{2} S_{4}$,

(ii) if $S_{1}, S_{2} \in \mathcal{S}$, then there exist $S_{3}, S_{4} \in \mathcal{S}$ such that $S_{1} S_{2}=S_{3} S_{1}$ $=S_{2} S_{4}$,

then the set $G=\bigcap_{S \in S} \mathrm{Cl}(S S)$ is a nonvacuous topological group.

If we order the semigroup $S$ by saying that $S_{1}>S_{2}$ whenever there is an $S_{3}$ such that $S_{1}=S_{2} S_{3}$, then $S$ is a directed set and $G$ is its collection of cluster points.

THEOREM 5. With the same hypotheses as for Theorem 4, if $S \in S$ and $G \in G$, then $S G \in G$ and $G S \in G$.

Our main theorem is the following.

THEOREM 6. Let. S be a totally bounded multiplicative semigroup in $\varepsilon$, which satisfies the pseudo-commutative laws (i) and (ii) of Theorem 4, then $S$ is an ergodic semigroup and every element of $X$ is ergodic.

Proof. We first show that $\bar{S}$ is a topological semigroup. Let $A$ and $B$ be elements of $\bar{S}$. We may choose directed sets $\left\{A_{\alpha} \mid \alpha \in \Delta\right\}$ and 
$\left\{B_{\alpha} \mid \alpha \in \Delta\right\}$ in $S$, so that $A_{\alpha} \in A+V_{\alpha}$ and $B_{\alpha} \in B+V_{\alpha}$. Then, $\left\{A_{\alpha} B_{\alpha} \mid \alpha \in \Delta\right\}$ is a directed set in $S$ which converges to $A B$ because, given $V_{\gamma}$, we may choose $V_{\beta}$ so that $\bar{S} V_{\beta} \subseteq V_{\gamma} / 2$, and $V_{\beta} B \subseteq V_{\gamma} / 2$. Then for $\alpha>\beta$ we have that

$$
\begin{aligned}
A_{\alpha} B_{\alpha}-A B & =A_{\alpha} B_{\alpha}-A_{\alpha} B+A_{\alpha} B-A B \\
& \in A_{\alpha} V_{\alpha}+V_{\alpha} B \\
& \subseteq\left(V_{\gamma}+V_{\gamma}\right) / 2 \subseteq V_{\gamma} .
\end{aligned}
$$

The set $\bar{S}$ is therefore a topological semigroup.

The semigroup $\bar{S}$ is compact because it is a totally bounded closed set in a boundedly complete topological linear space. By Theorem 4 , the set $G=\bigcap_{S \in S} \mathrm{Cl}(S S)$ is a topological group. By Theorem 3 and because $G \subseteq \bar{S}$, there is an operator $P \in \mathrm{Cl}(\operatorname{con} G)$ such that $P G=P$ $=G P$ for all $G \in G$. Then by Theorem 5 , if $S \in \mathcal{S}$ and $G \in G$, then $S G$ and $G S$ are in $G$ and

$$
S P=S(G P)=(S G) P=P=P(G S)=(P G) S=P S .
$$

We may therefore put $P=T_{\gamma}$ for all $\gamma$ and we obtain a trivial directed set, satisfying the conditions required in order that $\mathcal{S}$ should be an ergodic semigroup.

Finally if $x \in X$, then $y=P x \in \mathrm{Cl}((\operatorname{con} S) x)$ and $S y=S P x=P x=y$ for all $S \in \mathcal{S}$, so we have also that every element of $X$ is ergodic.

We may even say a little more about the operator $P$, as in the following theorem.

THEOREM 7. Under the hypotheses of Theorem 6, the following statements are equivalent.

(1) $P \in G$.

(2) $P=E$, the identity of $G$.

(3) $G$ consists of one element.

(4) If $S$ is directed by saying that $S_{1}>S_{2}$ whenever $S_{1}=S_{2} S_{3}$, then $S$ converges in $\varepsilon$.

(5) The directed set $\mathcal{S}$ converges in $\varepsilon$ to $E=P$.

Proof. (1) implies (2), because $P^{2}=P$ and a group can have only one idempotent element which is the identity.

(2) implies (3), because $G=E G=P G=P=E$ for all $G \in G$.

(3) implies (4), because the directed set $\mathcal{S}$ in the compact space $\bar{\delta}$ has a unique cluster point $E$, so that it converges to $E$.

(4) implies (5), because if the directed set $\delta$ converges, then from the definition of $G$ its limit is $E$ and $G$ reduces to one element $E$. Thus $P \in \mathrm{Cl}$ (con $G)=E$.

(5) implies (1) easily. 


\section{REFERENCES}

1. L. Alaoglu and G. Birkhoff, General ergodic theorems, Ann. of Math. vol. 41 (1940) pp. 293-309.

2. M. M. Day, Ergodic theorems for Abelian semigroups, Trans. Amer. Math. Soc. vol. 51 (1942) pp. 399-412.

3. - Means for the bounded functions and ergodicity of the bounded representations of semigroups, Trans. Amer. Math. Soc. vol. 69 (1950) pp. 276-291.

4. W. F. Eberlein, Abstract ergodic theorems, Trans. Amer. Math. Soc. vol. 67 (1949) pp. 217-240.

5. S. Kakutani, Two fixed point theorems concerning bicompact convex sets, Proc. Imp. Acad. Tokyo vol. 14 (1938) pp. 242-245.

6. J. E. L. Peck, The embedding of a topological semigroup in a topological group and its generalizations, unpublished.

7. K. Yosida, Asymptotic almost periodicities and ergodic theorems, Proc. Imp. Acad. Tokyo vol. 15 (1939) pp. 255-259.

8. D. H. Hyers, Linear topological spaces, Bull. Amer. Math. Soc. vol. 51 (1945) pp. 1-21.

9. A. E. Taylor, The weak topologies of Banach spaces, Proc. Nat. Acad. Sci. U.S.A. vol. 25 (1939) pp. 438-440.

10. J. von Neumann, On complete topological spaces, Trans. Amer. Math. Soc. vol. 37 (1935) pp. 1-20.

11. G. Birkhoff, Moore-Smith convergence in general topology, Ann. of Math. vol. 38 (1937) pp. 39-56.

12. W. Maak, Eine neue Definition der fastperiodischen Funktionen, Abh. Math. Sem. Hansischen Univ. vol. 11 (1936) pp. 240-244.

13. N. Bourbaki, Topologie générale, chaps. 1 and 2, Paris, 1940.

14. J. W. Tukey, Convergence and uniformity in topology, Annals of Mathematics Studies, no. 2, 1940.

15. E. Hille, Functional analysis and semi-groups, Amer. Math. Soc. Colloquium Publications, vol. 31, 1948.

YALE UNIVERSITY 